\title{
Incertidumbre en la determinación del Porcentaje Volumétrico de Bloques de BIMrocks/BIMsoil a partir de información unidimensional
}

\author{
Alfonso Mariano Ramos-Cañón ${ }^{1 *}$, Lady Carolina Castro-Malaver², Nelly Vanessa Padilla-Bello², \\ Carlos Alberto Vega-Posada ${ }^{3}$
}

doi: http://dx.doi.org/10.18273/revbol.v42n1-2020004 (c) (1)

Forma de citar: Ramos-Cañón, A.M., Castro-Malaver, L.C., Padilla-Bello, N.V., y Vega-Posada, C.A. (2020). Incertidumbre en la determinación del Porcentaje Volumétrico de Bloques de BIMrocks/BIMsoil a partir de información unidimensional. Boletín de Geología, 42(1), 69-80. doi: 10.18273/revbol.v42n1-2020004.

\section{RESUMEN}

La caracterización mecánica de depósitos de materiales heterogéneos (BIMrocks/BIMsoil - Block in Matrix) requiere del conocimiento de la cantidad de bloques inmersos en una matriz débil (Proporción Volumétrica de Bloques, PVB). La aleatoriedad en el proceso de formación de dichos depósitos impide obtener información unívoca mediante estudios geotécnicos convencionales.

Es posible reconocer que a medida que aumenta el número y la profundidad de las perforaciones, se disminuye la incertidumbre en la determinación de la PVB, sin embargo, la cuantificación de la disminución de la incertidumbre no ha sido abarcada de manera comprensiva. La determinación de la PVB en materiales BIMrock/BIMsoil tiene asociada una incertidumbre que se ha estudiado con modelos físicos y analíticos. Dichos modelos no consideran la influencia de factores como el tamaño, forma y orientación de los bloques, que simultáneamente inciden en la cuantificación de la incertidumbre en la PVB.

En este trabajo se desarrolló un algoritmo y se implementó computacionalmente para analizar la influencia de la longitud y el número de las perforaciones, junto con la forma y orientación de los bloques, sobre el nivel de incertidumbre en la determinación de la PVB. Los resultados sugieren que a mayor investigación del subsuelo (número y longitud de perforaciones), independiente de la forma, tamaño y orientación de los bloques, se disminuye la incertidumbre epistémica en la PVB. Sin embargo, dicha incertidumbre tiene un límite inferior que obedece a la naturaleza aleatoria propia de la formación del material BIMrock/BIMsoil.

Palabras clave: Incertidumbre; BIMrock/BIMsoil; proporción volumétrica de bloques; caracterización unidimensional.

\section{Uncertainty in the determination of the volumetric block proportion of BIMrocks/ BIMsoils from one-dimensional information}

\begin{abstract}
The mechanical characterization of the deposits of heterogeneous materials (BIMrocks/BIMsoil - Block in Matrix) requires the knowledge of the number of embedded blocks within a weaker matrix (Proportion of Volumetric Blocks, PVB). The randomness in the process of formation of such deposits does not allow to obtain univocal information through the conventional geotechnical survey.

It is possible to recognize that as the number and depth of the borehole increase, the uncertainty of the determination of the PVB decrease. However, the quantification of the decrease of uncertainty has not been fully covered. The determination of the PVB in materials BIMrock/BIMsoil has associated an uncertainty that have been studied with physical and analytical models. Such models do not consider the influence of factors like the size, shape and the blocks orientation, that simultaneously affect in the quantification of the uncertainties in the PVB.

In this work a computational algorithm has been developed and implemented to analyze the influence of the length and number of borehole, the shape and orientation of the blocks, on the uncertainty level of the determination of the PVB. The results suggest that further investigations of the subsoil (number and length of the perforations), independently of the shape, size and orientation of the blocks, the less is the epistemic uncertainty in the PVB. Nevertheless, it has been found that such uncertainty has an inferior limit that obeys the randomly natural formation of the material BIMrock/BIMsoil.
\end{abstract}

Keywords: Uncertainty; BIMrock/BIMsoil; volumetric block proportion; one-dimensional characterization.

${ }^{1}$ Universidad Nacional de Colombia, Bogotá, Colombia. (*) amramosc@unal.edu.co

${ }^{2}$ Universidad Militar Nueva Granada, Bogotá, Colombia. carolinacastroing@gmail.com, padillavane.vp@gmail.com

${ }^{3}$ Universidad de Antioquia, Medellín, Colombia. carlosa.vega@udea.edu.co 


\section{INTRODUCCIÓN}

El desarrollo de la infraestructura civil es afectado por la variabilidad y la complejidad geológica del suelo, la cual genera la necesidad de conocer y comprender el medio en el que se van a emplazar los proyectos. La aleatoriedad natural en el proceso de formación de las estructuras geológicas del suelo hace que la ingeniería enfrente materiales heterogéneos que, aunados a los métodos convencionales de exploración geotécnica, repercute en la dificultad de la estimación de sus propiedades físicas y mecánicas.

Los depósitos de materiales heterogéneos (BIMrocks/ BIMsoil - Block in Matrix, por sus siglas en inglés) se pueden definir como una mezcla de bloques geotécnicamente significativos embebidos en una matriz de suelo más débil con contraste mecánico entre bloques y matriz (Medley, 1994). Ejemplos de este tipo de depósitos son coluviones (Reneau y Dietrich, 1991), morrenas (Fernández y Ferrando, 2018), depósitos de brecha (Kahraman et al., 2015) y depósitos fluvioglaciares (Manchego y Peña, 2015).

Varios autores han mostrado que las propiedades mecánicas de los BIMrocks/BIMsoils dependen de la Proporción Volumétrica de Bloques (PVB) (Lindquist y Goodman, 1994; Sonmez et al., 2004, 2006; Kahraman y Alber, 2006; Napoli et al., 2018). La PVB se define como la relación entre el volumen de los bloques y el volumen total del depósito. La estimación directa de la PVB es difícil en la mayoría de los casos. Esta se puede determinar con base en información de carácter unidimensional (perforaciones, sondeos) (Tien et al., 2010), bidimensional (mapeo geológico en zonas expuestas de corte, análisis de fotografías) (Tien et al., 2011; Colmenares et al., 2018), tridimensional (análisis granulométricos en laboratorio o in situ) (Goodman y Ahlgren, 2000; Sonmez et al., 2004; Xu et al., 2007) o indirectos (relación con velocidad de propagación de ondas, densidad) (Kahraman et al., 2015; Mahdevari y Maarefvand, 2017).

Uno de los métodos más usados para hacer la investigación del subsuelo son las perforaciones. En este caso, se tiene información en una dimensión, a menudo vertical, del tipo de geomaterial. Cuando se realizan perforaciones en un depósito tipo BIMsoil/ BIMrock, se espera que la perforación atraviese bloques y que la información de la longitud de bloques que fue atravesado por la perforación sea tenida en cuenta como insumo para poder determinar el volumen de los bloques y la PVB. Medley y Goodman (1994) proponen que la
PVB puede ser supuesta estereológicamente equivalente a la Proporción Lineal de Bloques (PLB), donde PLB se define como la relación entre la longitud acumulada que atraviesa los bloques y la longitud total de la perforación. Es decir, a partir de un método para tener información en una dimensión se obtiene información tridimensional. Dependiendo de los métodos de perforación, de la consistencia de los componentes del depósito, del tipo de muestreador y de la broca, se puede tener información confiable de la longitud acumulada que atravesó los bloques embebidos en el depósito.

Se reconoce que a medida que se tiene mayor densidad de información (número y profundidad de perforaciones), la determinación de la longitud de penetración de los bloques es más representativa y por ende la estimación de la PVB tiene menor incertidumbre. Sin embargo, la cuantificación de la incertidumbre en la determinación de la PVB a partir de perforaciones no ha sido abordada de manera exhaustiva.

En este trabajo se retoman las variables que otros autores establecen como representativas de la incertidumbre en la determinación de la PVB y se consideran de forma simultánea para analizar su interrelación y cuantificar dicha incertidumbre en función de aspectos que pueden ser controlados en campo como el número y profundidad de las perforaciones. Se plantea y codifica un algoritmo para la generación de muestras cúbicas de una matriz débil con bloques embebidos de diferentes tamaños y formas, ubicados de manera aleatoria. A los especímenes se le realizan muestreos unidimensionales (perforaciones) con diferentes espaciamientos y profundidades. Como resultado, se cuantifica la incertidumbre asociada a la estimación de la PVB de un BIMrock/BIMsoil en términos del Coeficiente de Variación (CV) considerando la influencia de la profundidad y la cantidad de perforaciones en muestreos unidimensionales, así como el tamaño, forma y orientación de los bloques. Se encuentra que las variables que tienen la mayor influencia en la determinación de la incertidumbre de la PVB son el espaciamiento y la profundidad del muestreo, sobre el tamaño de los bloques, forma de los bloques y tamaño del espécimen. Los resultados se comparan con trabajos previos y se propone un límite inferior de la incertidumbre en la PVB que no puede ser disminuido aunque se incremente el número y la longitud de las perforaciones. Lo anterior, debido a la naturaleza aleatoria propia de la formación del material BIMrock/BIMsoil (incertidumbre aleatoria).

El documento se estructura de la siguiente manera: inicialmente, se presentan las variables que se tuvieron 
en cuenta para la generación de las muestras numéricas, posteriormente se presenta el procedimiento para el muestreo unidimensional y su relación con la PVB. En la sección de análisis de resultados y conclusiones se enfatiza en aspectos esenciales en la determinación de la incertidumbre de la PVB en función de la profundidad y el espaciamiento de las perforaciones.

\section{ANTECEDENTES}

El trabajo desarrollado por Medley (1997), permite dar cuenta de una primera estimación de la incertidumbre, mediante elaboración manual de especímenes de BIMrocks y de muestreo unidimensional. Medley (1997) dibujó líneas sobre el área simulando núcleos de perforación para calcular la PLB y concluyó que mientras el total de la longitud de muestreo (o la longitud de la línea dibujada) y la proporción volumétrica aumentan, la incertidumbre disminuye. Dicho trabajo se desarrolló con modelos físicos por lo que no tuvo en cuenta la influencia de variables como la distribución granulométrica, la forma y la orientación de los bloques.

Posteriormente, Tien et al. (2010, 2011), mediante modelos numéricos y soluciones analíticas, cuantificaron la incertidumbre en la PVB de un BIMrock/BIMsoil a partir de información unidimensional en muestras con bloques de un solo tamaño representativo. Medley (2002) reconoce que la incertidumbre en la estimación de la PVB depende de muchos factores, entre los más importantes menciona la forma de los bloques, la distribución de los tamaños de los bloques, la PVB, la orientación de los bloques y la longitud total de la muestra del BIMrock/BIMsoil.

\section{METODOLOGÍA}

\section{Generación de las muestras unidimensionales}

Con el objetivo de analizar la incertidumbre asociada a la determinación de las PVB en un BIMrock/BIMsoil mediante exploración unidimensional se desarrolló un algoritmo en el que se identifican las siguientes fases: asignación de las variables a estudiar, generación de los especímenes, muestreo unidimensional y tratamiento de datos. Para la creación de los especímenes, se conformaron muestras cúbicas que mediante la asignación de las variables Longitud Característica (Lc), PVB, tamaño, forma y ubicación de los bloques, y su combinación permitieron modelar las condiciones de las muestras numéricas representativas de un BIMrock/BIMsoil.
El tamaño de cada muestra cúbica se determinó a partir de Lc, definida por Medley (1994) como cualquier dimensión observable en un BIMrock/BIMsoil que permita establecer la escala de trabajo. Para las muestras cúbicas se asignaron Lc de 0,$5 ; 1 ; 2 ; 3 ;$ y 4 unidades de longitud (Figura 1A). Cada espécimen tiene una PVB determinada en un rango de $4 \%$ al $19 \%$ en función de la distribución uniforme de tamaños de los bloques, su distribución dentro de cada espécimen y su forma. El rango obedece a que la mayor variabilidad en los resultados del incremento de la resistencia mecánica está aproximadamente entre 5\% al 20\% (Sonmez et al., 2006).

Para cada espécimen se generaron bloques de diez tamaños distribuidos de manera equitativa dentro del rango del 5\% Lc hasta el 75\% Lc. El rango de tamaño obedece a que cuando el BIMrock/BIMsoil tiene bloques con tamaño menor al $5 \%$ Lc, su comportamiento mecánico se debe a las propiedades de la matriz y para BIMrock/BIMsoil con bloques cuyo tamaño son mayores al $75 \% \mathrm{Lc}$, el comportamiento se debe a las propiedades de los bloques (Medley, 1994). En el rango de tamaños de bloques entre el 5\% Lc y el $75 \%$ Lc se encuentra el comportamiento mecánico intermedio entre la matriz débil y los bloques.

Para tener en cuenta la forma de los bloques, se consideraron esferas y elipsoides, variando para los bloques de cada espécimen las dimensiones en sus ejes mayor, intermedio y menor $(\mathrm{x}, \mathrm{y}, \mathrm{z})$ de tal forma que se multiplicaron una, dos y tres veces la dimensión del bloque (el radio) establecida por la distribución de tamaños dentro del rango del comportamiento mecánico del BIMrock/BIMsoil. Las formas se agruparon de acuerdo a la relación de ejes $(\mathrm{x}, \mathrm{y}, \mathrm{z})$ : esferas 1:1:1 (relación de ejes 1:1:1, sin modificar el radio), elipsoides (relación de ejes diferentes a 1:1:1); en total se obtuvieron ocho formas (Figura 1B).

Los bloques se ubicaron dentro de la muestra de manera aleatoria siguiendo una función de distribución de probabilidad uniforme, generados dentro del código escrito para la modelación, asegurando que la ubicación del centroide de cada bloque estuviera contenida dentro de la muestra y que no se presentara intersección entre bloques de un mismo espécimen. La ubicación aleatoria se realizó de forma independiente para cada bloque del espécimen. En la Figura 2 se presenta un ejemplo para dos especímenes de longitud característica $\mathrm{Lc}=0,5$ con PVB de 4,4\% y $9,9 \%$, con diferentes relaciones de esbeltez de los bloques a. $\mathrm{x}, \mathrm{y}, \mathrm{z}=3,2,3$ b. $\mathrm{x}, \mathrm{y}, \mathrm{z}=2,2,3$. 

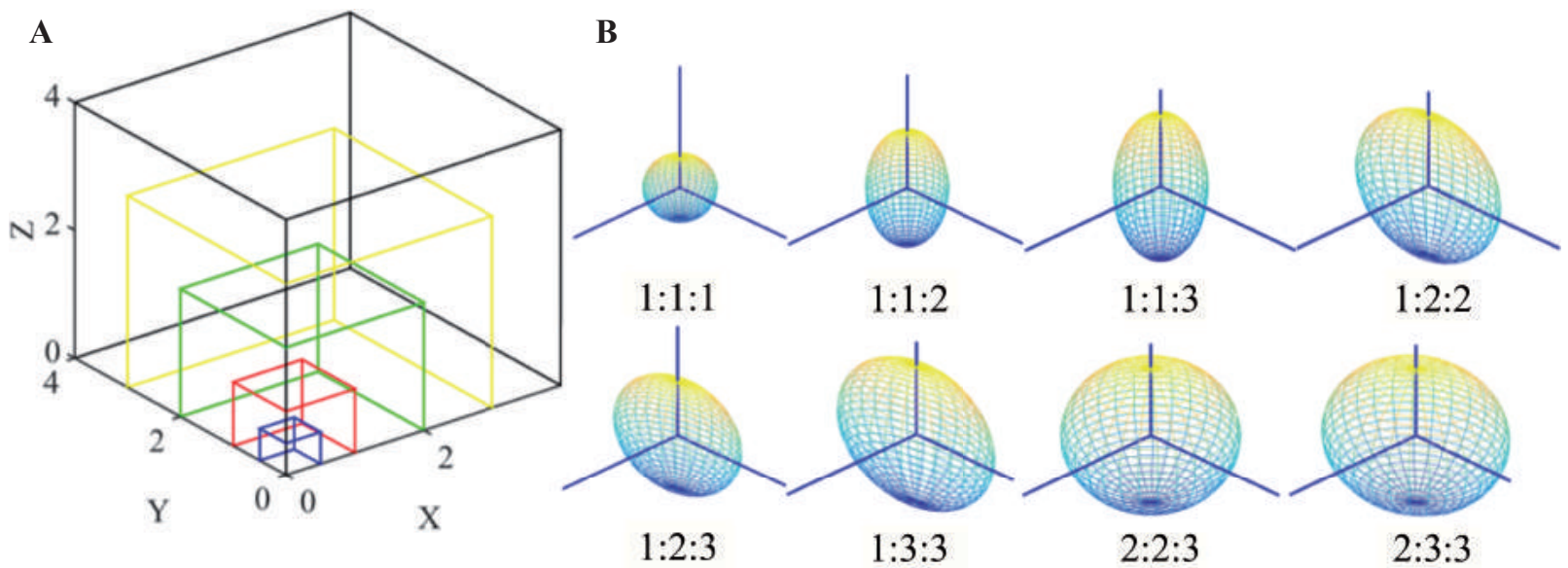

Figura 1. A. Especímenes cúbicos de dimensiones $\mathrm{Lc}=0,5 ; 1 ; 2 ; 3$ y 4 unidades. B. Formas de los bloques en función de la relación entre las dimensiones de los ejes mayor, intermedio y menor de los elipsoides.

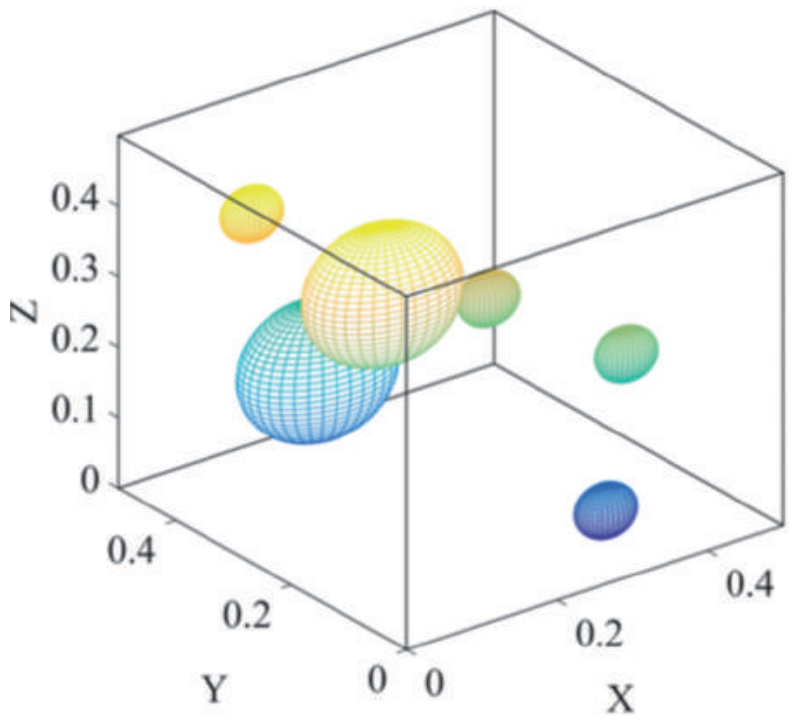

A

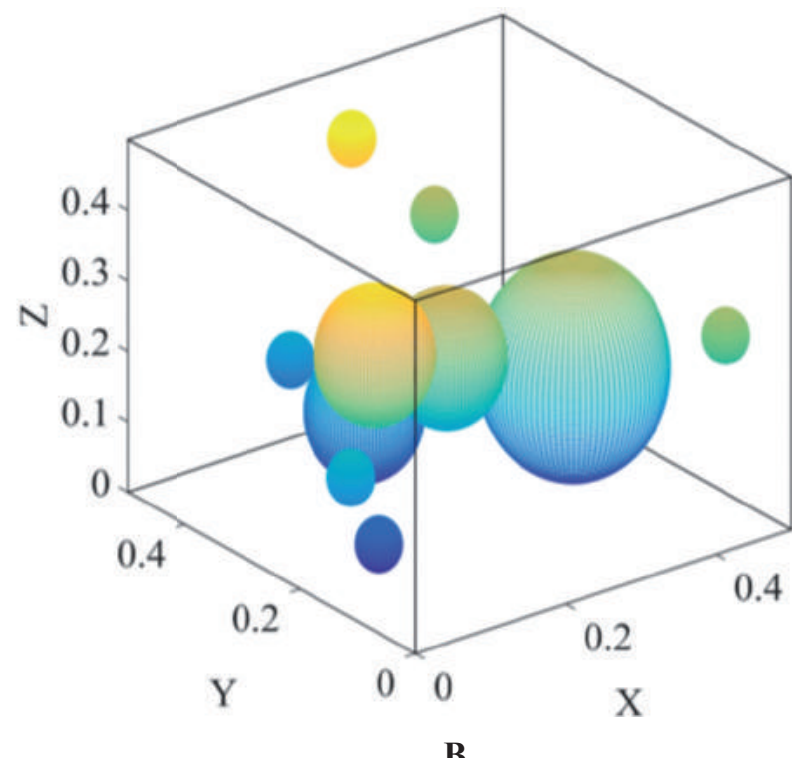

B

Figura 2. Especímenes numéricos generados para este estudio A. PVB $=4,4 \%$; Lc $=0,5$; esbeltez $x, y, z=3,2,3$; número de bloques $=6$ y $\mathbf{B} . \mathrm{PVB}=9,9 \%$; $\mathrm{Lc}=0,5$; esbeltez $\mathrm{x}, \mathrm{y}, \mathrm{z}=2,2,3$; número de bloques $=10$.

La distribución de tamaños de bloques que se generaron numéricamente para todos los especímenes presentan un coeficiente de uniformidad $\mathrm{Cu}=4,276$ y coeficiente de curvatura $\mathrm{Cc}=1,248$ (Figura 3). La distribución del tamaño de los bloques corresponde a una granulometría medianamente uniforme y bien gradada, según los criterios de clasificación de la Unified Soil Classification System (USCS): son curvas uniformes con valores de $\mathrm{Cu}>3$ y granulometrías bien gradas con $1<\mathrm{Cc}<3$ (Das, 2001).
La Figura 3 presenta las curvas granulométricas de los bloques con los que se generaron las muestras numéricas para las cinco diferentes dimensiones de espécimen ( $\mathrm{Lc}=0,5 ; 1 ; 2 ; 3$ y 4 unidades de longitud). Las unidades de las abscisas de la Figura 3 corresponde a unidades de longitud para una escala de trabajo dada por Lc. 


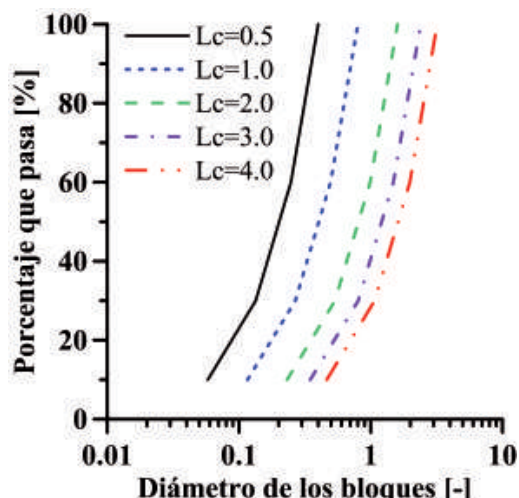

Figura 3. Distribución granulométrica de los bloques con los que se generaron los especímenes cúbicos numéricos para longitudes de muestra $\mathrm{Lc}=0,5 ; 1 ; 2 ; 3$ y 4 . Diámetro de los bloques en unidades de longitud.

En la Figura 4 se presenta a manera de ejemplo, las tres diferentes orientaciones que pueden tener los bloques en función de la relación entre los ejes mayor, intermedio y menor de los elipsoides.
A

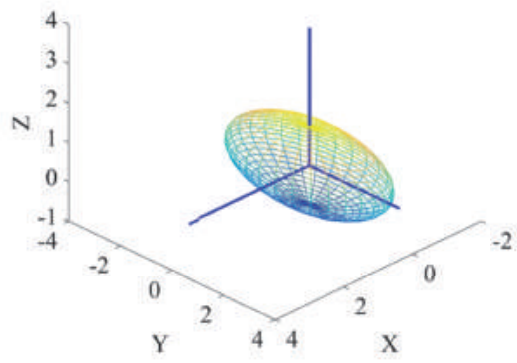

B

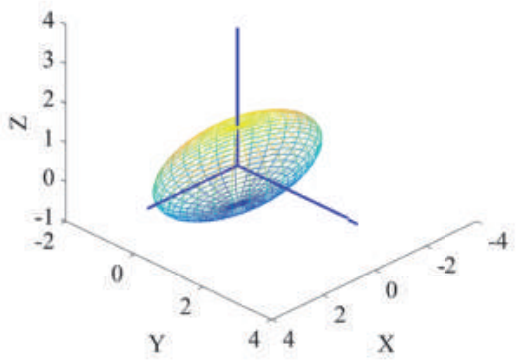

C

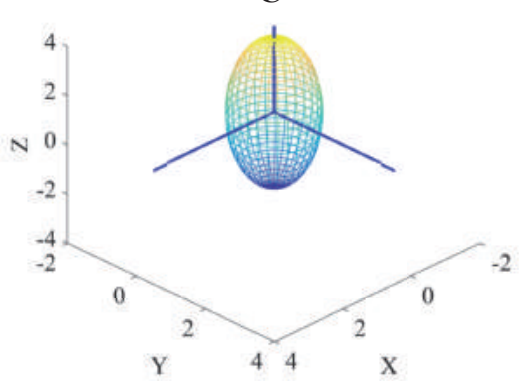

Figura 4. Orientación de los bloques en función de la relación entre los ejes mayor, intermedio y menor de los elipsoides. A. x, $y, z=1,1,3, \mathbf{B} . x, y, z=3,1,1, \mathbf{C} . x, y, z=1,3,1$.

\section{Muestreo unidimensional}

Una vez generadas las muestras numéricas, se realiza muestreo unidimensional vertical a cada espécimen con diferentes profundidades equivalentes al 30\%, $60 \%$ y $100 \%$ de Lc (Figura 5). La profundidad de
$100 \%$ Lc penetra la totalidad de la muestra. El muestreo se realizó a través de mallas cuadradas conformadas por 4 (2X2), 9 (3X3), 16 (4X4) y 25 (5X5) perforaciones con una ubicación equidistante. La distancia entre perforaciones para cada malla equivale al 33,3\%, 25\%, $20 \%$ y $16,7 \%$ de Lc.
A

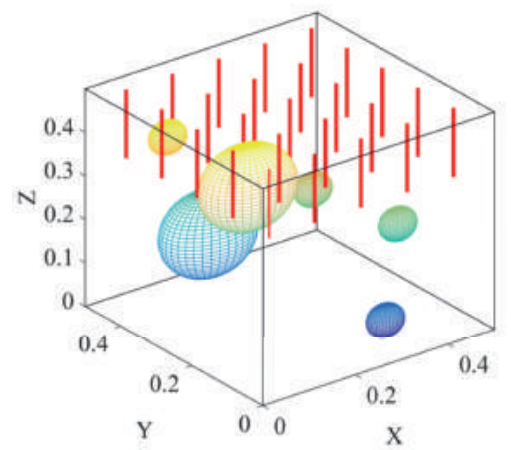

B

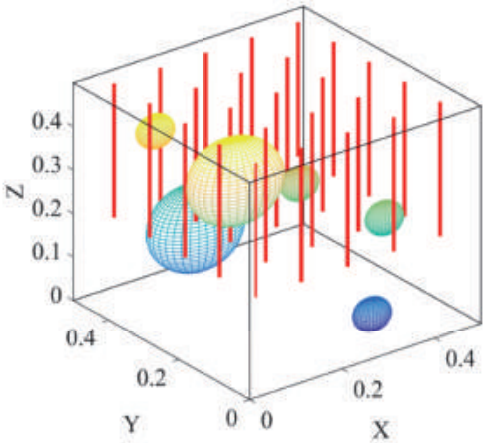

C

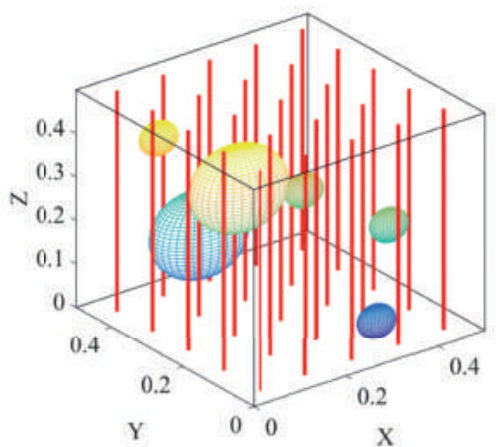

Figura 5. Especímenes con $\mathrm{Lc}=0,5 ; \mathrm{PVB}=4,4 \%$; esbeltez $\mathrm{x}, \mathrm{y}, \mathrm{z}=3,2,3$; número de bloques $=6$. Muestreo con malla de 25 perforaciones unidimensionales equivalente a $16,7 \%$ Lc de espaciamiento, con profundidades de muestreo de A. $30 \% \mathrm{Lc}, \mathbf{B}$. $60 \%$ Lc y C. $100 \%$ Lc. 
Para cada muestreo unidimensional, se calcula la relación entre la longitud acumulada de las intersecciones de los bloques y longitud total de esta misma, a lo que se denomina PLB expresado en porcentaje (Figura 6). Este valor es el que se utiliza para estimar la PVB pasando información de una dimensión PLB a información volumétrica con lo que necesariamente se genera incertidumbre en la estimación. En la Figura 6 se presenta un espécimen construido con una PVB de 4,4\%. Se presenta además el resultado de obtener la PLB con una perforación que penetra la totalidad del bloque (PLB=15,08\%).
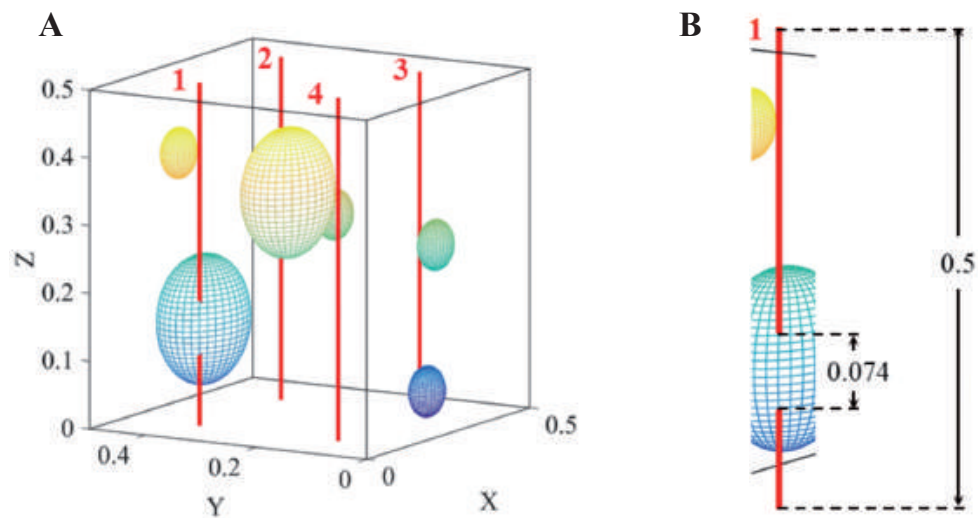

Figura 6. A. Espécimen cúbico con 0,5 de longitud característica (Lc), bloques esféricos y PVB=4,4\%. 4 perforaciones al $100 \%$ de profundidad $(100 \% \mathrm{Lc})$. B. Bloques intersectados con una perforación de profundidad 100\% Lc. PLB es de 15,08\%.

\section{RESULTADOS}

Cuando se realiza una perforación en un material tipo BIMsoil/BIMrock, se espera obtener información de la PVB con base en información de los núcleos de material de bloque, es decir, de la PLB. Se reconoce el hecho que el valor de la PLB es diferente de la PVB. En la Figura 7 se presenta la diferencia en valor absoluto entre la PVB con que se construyó cada espécimen y el promedio de la PLB de las perforaciones, normalizado por la PVB, en función de la PVB para diferentes mallas $(2 \mathrm{X} 2,3 \mathrm{X} 3,4 \mathrm{X} 4$ y $5 \mathrm{X} 5)$. A esta expresión se le denominó ErrorPVB (Ecuación 1).

$$
\text { ErrorPVB }=\frac{\left|P V B-\frac{\sum_{i=1}^{n} P L B_{i}}{n}\right|}{P V B}
$$

Donde $P L B_{i}$ es la proporción lineal de bloques de la perforación $i$-esima y $n$ es el número de perforaciones que fueron generadas en cada espécimen. El ErrorPVB es adimensional.

La Figura 7 se generó con perforaciones que atravesaban la totalidad del espécimen $(\mathrm{Lc}=100 \%)$.

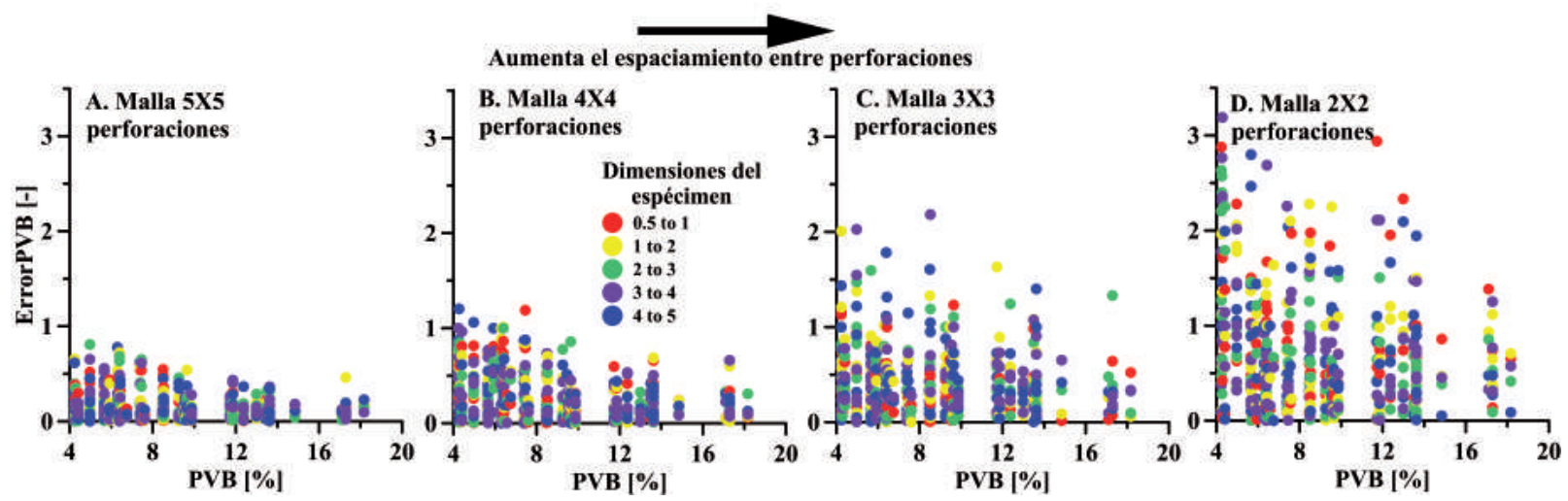

Figura 7. Diferencia entre la PVB y la PLB normalizado para diferentes dimensiones de espécimen. Malla de perforaciones A. $5 X 5$, B. 4X4, C. 3 X3 y D. 2X2. Longitud de penetración de la perforación de $100 \%$ Lc. 
Por otro lado, si se hace una perforación en un espécimen de PVB determinado, se obtiene un valor de PLB. Cada vez que se realizan perforaciones adicionales, se obtendrán diferentes valores de PLB con el que se intenta obtener el valor de la PVB y así se genera una incertidumbre su estimación.

La incertidumbre en la estimación de la PVB se realizó a través de la evaluación del coeficiente de variación del muestreo en una dimensión (COV). Para cada espécimen se realiza un número de perforaciones, se calculan las PLB, se determina la desviación estándar de la PLB y finalmente se calcula el COV dividiendo la desviación estándar entre el valor medio $(P L B)$ (Ecuación 2).

$$
C O V=\frac{\sigma_{P L B}}{\overline{P L B}}
$$

La Figura 8 presenta la evaluación de la incertidumbre en la PLB en términos del COV para valores de la PVB.
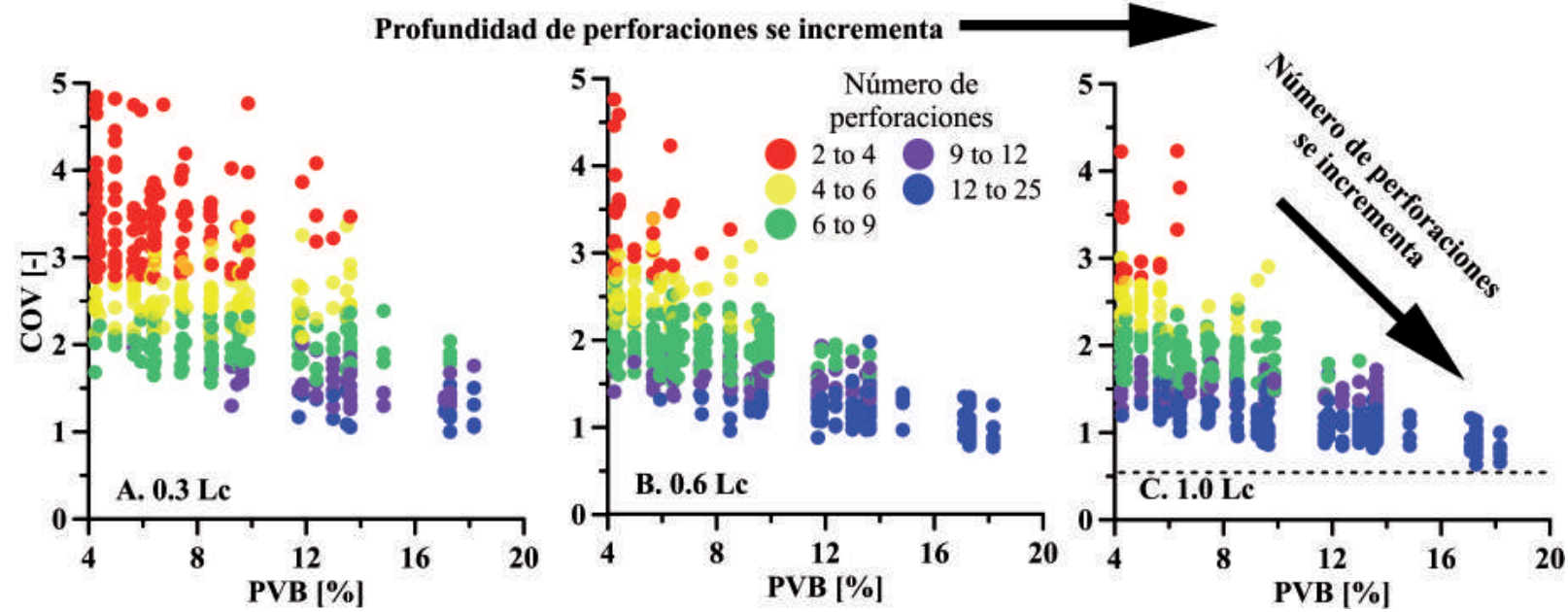

Figura 8. Coeficiente de variación (COV) de la Proporción Lineal de Bloques (PLB) en función del PVB de especímenes para diferentes longitudes de perforación A. $0,3 \mathrm{Lc} ;$ B. $0,6 \mathrm{Lc}$; C. 1,0 Lc. Malla de 5 X5 perforaciones equivalentes a espaciamiento de $16,7 \%$ Lc. Datos discriminados por número de perforaciones.

La Figura 8 presenta la influencia de la profundidad de perforación (Figura 8A) en relación con el tamaño del espécimen. $0,3 \mathrm{Lc}$ indica que las perforaciones para la Figura 8A llegaron hasta una profundidad de 0,3Lc. Para calcular el COV se tuvo en cuenta aquellos especímenes que tenían dos o más perforaciones que habían interceptado bloques.

En la Figura 7 se presentó el ErrorPVB para longitudes de perforación del $100 \%$ de Lc en cada una de las mallas usadas $(2 \mathrm{X} 2,3 \mathrm{X} 3,4 \mathrm{X} 4$ y $5 \mathrm{X} 5)$. Estas mallas son reflejo de la influencia de la distancia entre perforaciones, que equivalen al $33,3 \% ; 25 \% ; 20 \%$ y $16,7 \%$ de Lc. Un valor alto en las ordenadas en la Figura 7 indica un error en la estimación del PVB con base en el \% lineal mayor.

Se observa que, para un espaciamiento dado de las perforaciones, el nivel de error en la determinación de PVB se mantiene aproximadamente constante en función de la mismo PVB. Al comparar las diferentes mallas en la Figura 7, se obtiene que el error en la determinación de la PVB en términos de la PLB es mayor a medida que aumenta la separación entre perforaciones. Es decir, a más distanciamiento entre perforaciones se obtiene mayores niveles de error. En una malla de $5 \times 5$, cuyo espaciamiento entre perforaciones equivale a distancias de $16,7 \%$ de Lc, si la PVB es $17 \%$, el porcentaje PLB de las perforaciones puede arrojar valores entre $4 \%$ y $30 \%$. En una malla de $2 \mathrm{X} 2$, si la PVB es del 17\%, la PLB puede variar entre $10 \%$ y $45 \%$ dados los valores de diferencia absoluta normalizada en la Figura 7D de 1,6. La diferencia entre la PLB observada en una perforación con la PVB es eexacerbada a medida que la PVB es menor. 
Se presenta, además, que las dimensiones del espécimen no influyen en la diferencia absoluta normalizada de determinación de la PVB ya que no se aprecia ninguna tendencia o agrupación significativa.

La incertidumbre en la estimación de la PVB reflejada en el COV y presentada en la Figura 8, disminuye a medida que aumenta la PVB, pero esto se hace más notorio a medida que se tiene mayor número de perforaciones, es decir, a medida que hay más bloques en el espécimen, la incertidumbre en la estimación a través de perforaciones es menor. A medida que el espécimen tiene mayor cantidad de bloques, el volumen de bloques aumenta y por ende se obtiene un aumento en la posibilidad de interceptar los bloques, lo que redunda a su vez en más información y consecuentemente menor incertidumbre.

\section{DISCUSIÓN DE RESULTADOS}

De la Figura 8 también se obtiene que a medida que aumenta el número de perforaciones para inferir la PVB se disminuye la incertidumbre para cualquier profundidad de perforación. De manera similar se obtiene que a mayor profundidad de las perforaciones disminuye la incertidumbre en la estimación de la PVB. En ambos casos se infiere que, a mayor cantidad de información, ya sea por el incremento en el número de perforaciones o por mayor profundidad, se disminuye la incertidumbre en la estimación de la PVB.

Se observa además que existe un límite inferior en la incertidumbre de coeficiente de variación a pesar de la alta densidad en el muestreo. En la Figura 8C se presentan datos de $\mathrm{COV}$, malla de $5 \times 5$ (espaciamiento entre perforaciones equivalentes a $16,7 \%$ Lc). Lo anterior indica que a pesar de que se quiera disminuir la incertidumbre en la determinación de la PVB con el aumento del número de perforaciones, la incertidumbre no se puede anular totalmente. La incertidumbre epistémica disminuye con el aumento en la cantidad de información, pero la incertidumbre aleatoria permanece debido a la variabilidad inherente por la naturaleza propia de conformación de los BIMrock/BIMsoil. La asíntota en la Figura $8 \mathrm{C}$ para la incertidumbre aleatoria es del orden de $\mathrm{COV}=0,5(50 \%)$ para los mayores PVB. Se observa además que la incertidumbre epistémica en la PVB disminuye de casi $\mathrm{COV}=2(200 \%)$ (Figura $8 \mathrm{~A}$ ) a valores de $\mathrm{COV}=1(100 \%)$ (Figura $8 \mathrm{C}$ ).

El coeficiente de variación ha sido uno de los indicadores de la incertidumbre que se ha usado con frecuencia para variables aleatorias. Coeficientes de variación típicos de propiedades como la densidad puede ser del orden de $\mathrm{COV}=0,10$; otras propiedades geotécnicas pueden tener más incertidumbre como la permeabilidad que puede tener valores de $\mathrm{COV}=2 \mathrm{o}$ $\mathrm{COV}=3$ (Baecher y Christian, 2005). Por lo anterior, la incertidumbre aleatoria de la estimación de la PVB puede ser mayor a la de variables que típicamente se usan en análisis de confiabilidad.

La Figura 9 presenta la misma información de la Figura 8 , con la diferencia que los datos son discriminados por la relación de ejes de los bloques (Figura 9A) y por la longitud del lado del espécimen (Lc) (Figura 9B) para analizar la influencia que tiene la forma de los bloques y las dimensiones del espécimen en la incertidumbre de la PVB. Se observa que la información del COV no presenta tendencia a agruparse cuando se diferencia por las dimensiones del espécimen ni cuando se diferencia por la forma contenida en la relación de ejes para la conformación de los bloques. Diferente a lo que pasa en la Figura 8 donde claramente los datos se diferencian y agrupan por el número de perforaciones junto con una influencia marcada de la longitud de la perforación. Lo anterior indica que ni la longitud de la muestra, ni la forma de los bloques son factores que modulen la incertidumbre en la determinación de la PVB, mientras que tanto el número de perforaciones como la profundidad de muestreo es un aspecto que permite diferenciar (aumentar o disminuir) la incertidumbre en la estimación de la PVB.

Medley (1997) presenta estimaciones de incertidumbre de PVB mediante la simulación de perforaciones unidimensionales a especímenes elaborados manualmente con diferentes fracciones volumétricas representativas de un BIMsoil/BIMrock (se obtuvieron resultados de $\mathrm{COV}=60 \%$ para $\mathrm{PVB}=13 \%)$. El análisis se realizó con un número definido de muestras de laboratorio y la metodología propuesta no resulta de fácil replicabilidad, por lo que los resultados arrojados tienen utilidad limitada para ser generalizados.

La aproximación numérica acá presentada analiza la influencia de diferentes variables, tanto de los bloques (forma, tamaño, orientación) como del muestreo (profundidad y espaciamiento) en un escenario más cercano a la realidad a través de especímenes de tres dimensiones (cubos y elipsoides), conformados por volúmenes de bloques que son atravesados por líneas, que hacen las veces de perforaciones. Diferente al trabajo realizado por Tien et al. (2010) cuya aproximación es realizada por la generación de un espécimen numérico bidimensional atravesado por líneas. En 2011, Tien et 
al. (2011) avanzó en su aproximación para tener en cuenta la anisotropía de los ejes de los bloques a partir de incluir elipses en lugar de círculos para los bloques. A pesar de lo anterior, la aproximación sigue siendo de inferir información de 1D a partir de información de 2D para dar cuenta de un aspecto que es volumétrico. En contraste con lo que se realizó en el presente trabajo, donde se presenta una evaluación de la incertidumbre a partir de información de $1 \mathrm{D}$, con base en el análisis de información volumétrica, limitado siempre por la naturaleza de la modelación numérica, que resulta ser una simplificación de la realidad donde no se consideran en totalidad las variables que tienen influencia.

A pesar de las diferencias en las suposiciones de los trabajos desarrollados por Tien et al. $(2010,2011)$ con aquellas propuestas en el presente trabajo, la Figura 10 compara los resultados del COV en función del PVB.
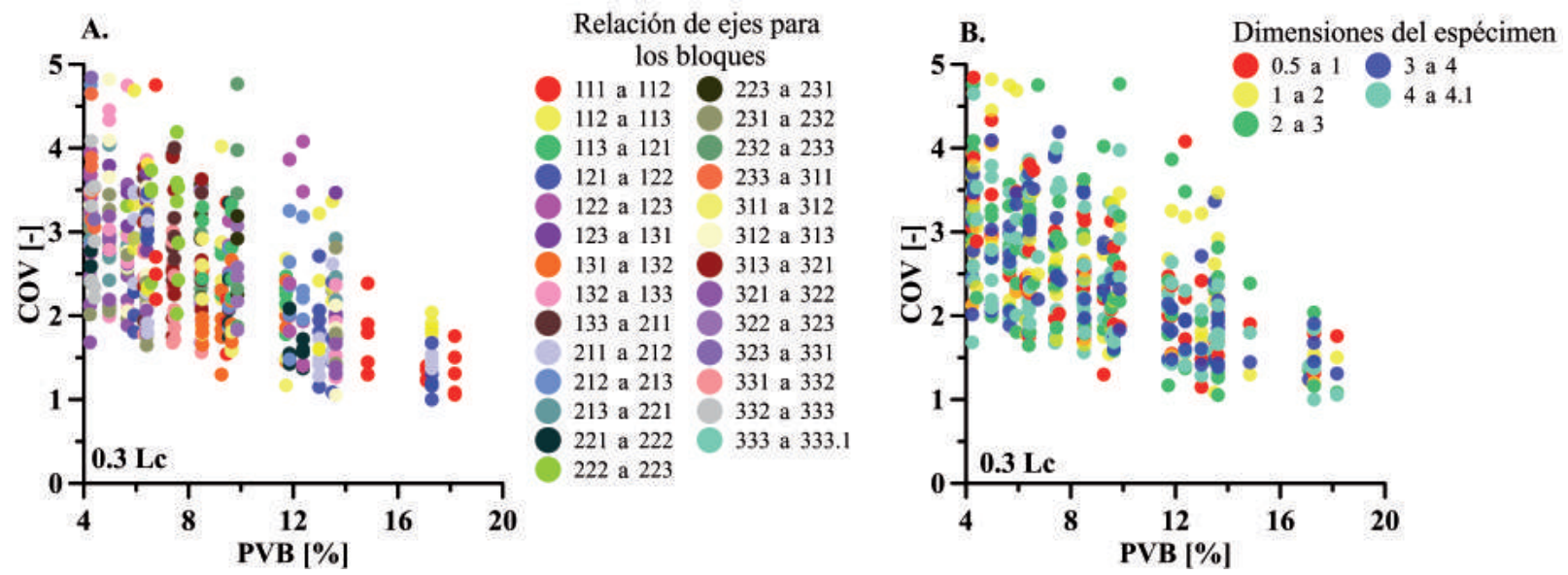

Figura 9. Coeficiente de variación de la proporción lineal de bloques en función del PVB de especímenes para longitudes de perforación 0,3Lc. Malla de 5X5 perforaciones equivalentes al espaciamiento de 16,7\% Lc. A. Datos discriminados por la relación de ejes para los bloques. B. Datos discriminados por dimensiones del espécimen.
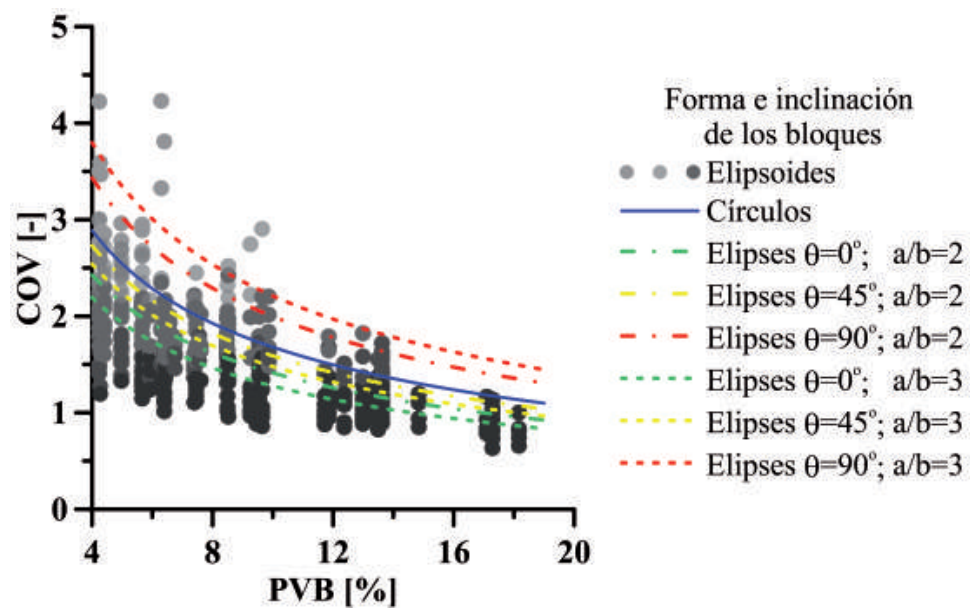

Figura 10. Comparación de la incertidumbre encontrada a través de información de bloques representados por elipses con diferentes grados de inclinación en un plano y relación de ejes a/b (propuesta de Tien et al., 2010, 2011) con respecto a los elipsoides con la misma relación de ejes en tres dimensiones e inclinación.

En la Figura 10, $\theta$ se refiere a la inclinación del eje mayor de los bloques representados por la elipse con respecto a la horizontal y a/b es la relación de ejes mayor y menor de las elipses (Tien et al., 2011). Se presenta además la línea que representa el COV en la determinación de la PVB para círculos, según lo propuesto por Tien et al. (2010). Se observa en general que la tendencia de la disminución de la incertidumbre es concordante en todas las aproximaciones. Del trabajo de Tien et al. (2010, 2011) se observa que a mayor PVB disminuye la incertidumbre, independiente de la forma de los bloques representados por elipses o círculos; similar a 
lo que se observó en la Figura 9A. Aunque en la Figura 9A no se evidencia ninguna tendencia en cuanto a la relación de ejes, para Tien et al. $(2010,2011)$ a mayor relación de esbeltez, se incrementa la incertidumbre para una misma inclinación y a mayor inclinación del eje de la elipse se incrementa la incertidumbre.

La evaluación de la incertidumbre en la determinación de la PVB que se propone en el presente trabajo mejora la aproximación de la solución analítica entregada por Tien et al. $(2010,2011)$ debido a que el análisis proviene de simulación de perforaciones de bloques embebidos en matriz fina de tipo elipsoides (3D). Es de esperar que los resultados entregados por Tien et al. $(2010,2011)$ difieran de la evaluación de la incertidumbre de la PVB dado que son aproximaciones disímiles (numérica, teórica) con suposiciones diferentes (los bloques son representados por elipses o círculos en Tien et al. (2010, 2011) en tanto que los bloques son representados por elipsoides en el presente trabajo). Dado lo anterior, se presenta un límite inferior a la estimación de la incertidumbre de la PVB en función de la misma PVB de aproximadamente COV=0,5 (50\%). En esta propuesta se contempla, por un lado, la variabilidad en la forma, orientación y tamaño de los bloques; y, por otro lado, el número y la longitud de las perforaciones para la determinación de la PVB.

De la información obtenida del arreglo $5 \times 5$, se analiza la influencia del número de perforaciones en la incertidumbre de la PVB con base en información unidimensional. La Figura 11 presenta la manera cómo varía el $\mathrm{COV}$ con el incremento en el número de perforaciones para diferentes PVB. Se observa que para valores bajos de PVB no se distingue una tendencia clara del COV (Figura 11C) con una alta dispersión en el COV en función de la PVB. Dicha variabilidad disminuye a medida que aumenta la PVB y se puede observar que a medida que aumenta el número de perforaciones, disminuye el COV. Es decir, la incertidumbre en la determinación de la PVB disminuye a medida que se tiene mayor cantidad de información. Se puede observar que el límite inferior del COV para altas PVB (Figura 11A) varía desde 1 a menos de 0,5 . Después de aproximadamente 10 perforaciones se puede establecer que dicho límite inferior tiende a volverse constante.
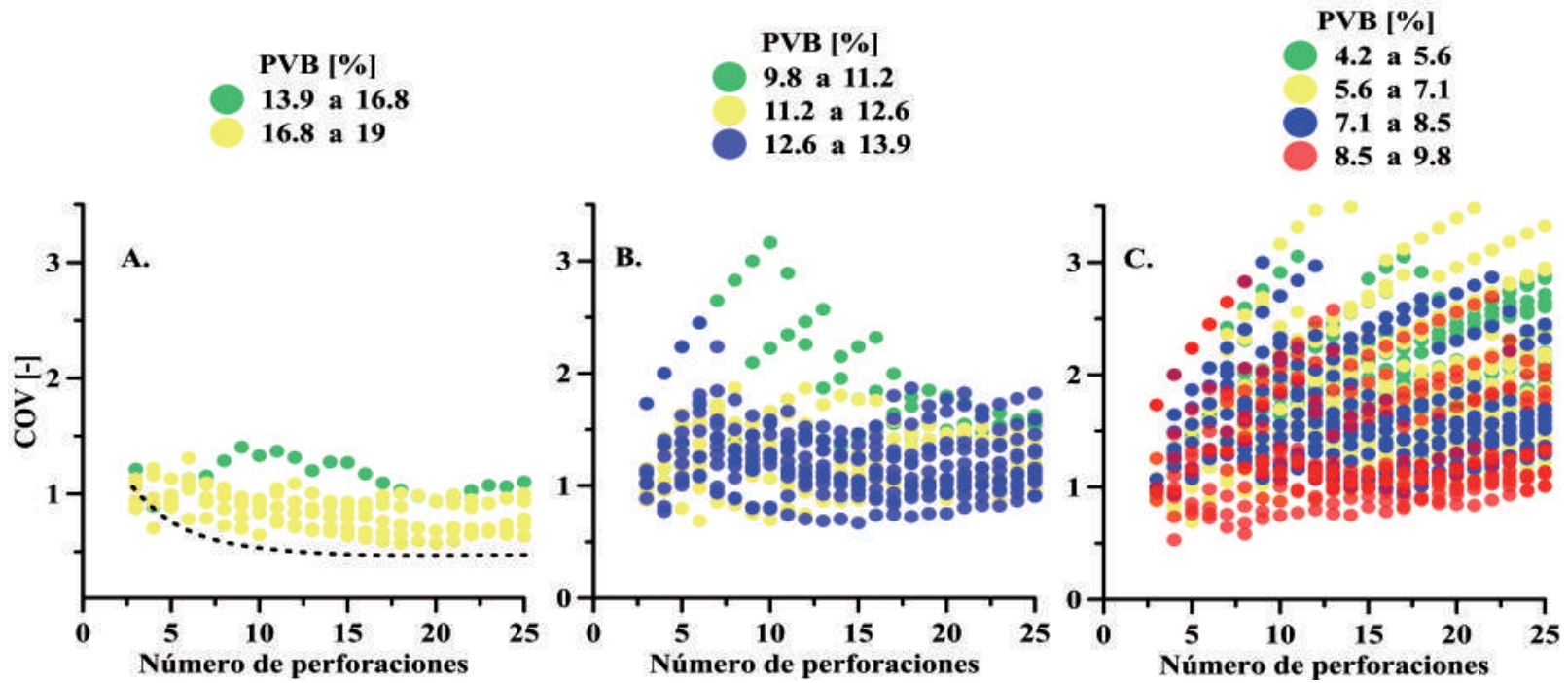

Figura 11. Coeficiente de variación de la proporción lineal de bloques en función del número de perforaciones para longitudes de perforación 1,0 Lc. Datos obtenidos de la malla de 5 X5 perforaciones equivalentes al espaciamiento de 16,7\% Lc. A. Altos PVB (13,9 y 19\%), B. Medio PVB (9,8 y 13,9\%), C. Bajo PVB (4,2 a 9,8\%).

La información contenida en la Figura 11A, sirve como referencia para establecer el impacto de incrementar el número de perforaciones en la incertidumbre en la determinación de la PVB, que a su vez se relaciona con las propiedades de resistencia del BIMrock/BIMsoil (Lindquist y Goodman, 1994; Sonmez et al., 2004, 2006; Kahraman y Alber, 2006; Napoli et al., 2018). Se sabe que, a medida que aumenta la incertidumbre en un parámetro que es proporcional a la resistencia, en este caso la PVB, aumenta la probabilidad de falla; a su vez, la probabilidad de falla es proporcional a los costos de falla (Baecher y Christian, 2005). Es decir, que con un análisis de confiabilidad se podría establecer la influencia del incremento del número de perforaciones en la disminución de los costos totales de un proyecto que esté emplazado en un BIMrock/ 
BIMsoil. Lo anterior, teniendo presente que los costos totales de un proyecto son los costos de construcción más los costos de falla, que a su vez son proporcionales a la probabilidad de falla.

\section{CONCLUSIONES}

El procedimiento propuesto en el presente trabajo mejora aproximaciones desarrolladas por anteriores autores debido a que la estimación de la incertidumbre se realiza con base en análisis de bloques en tres dimensiones, con la consideración de variables adicionales como la forma, tamaño y orientación de los bloques, el tamaño de los especímenes y el número y profundidad de las perforaciones.

Se presenta un error inherente en la estimación de la PVB con base en la PLB. Dichas diferencias son mayores a medida que se tiene menos información $y$ que la PVB es menor. Las diferencias pueden conllevar a que las estimaciones de la PVB puedan diferir en casos hasta en un factor mayor que 2 (COV>200\%).

Los resultados del procedimiento aplicado presentan valores de incertidumbre en términos del $\mathrm{COV}$, que pueden ser usados en análisis de confiabilidad para tener en cuenta, tanto la incertidumbre epistémica como la incertidumbre aleatoria en la determinación de la PVB. A pesar de las simplificaciones que conlleva la simulación numérica, se presentan resultados que pueden tomarse como referencia para análisis de confiabilidad donde se involucre la PVB.

Debido a que los resultados presentados son producto de modelación numérica, es necesario tener presente las limitaciones propias del ejercicio que han sido puestas en relieve a lo largo del artículo.

Se cuantifica la incertidumbre en la evaluación de la PVB. Se propone un valor mínimo de incertidumbre en el COV de 0,5 para los casos en donde se tiene un número significativo (mayor a 10) de perforaciones con longitudes de penetración en el depósito adecuadas.

Se encuentra que las variables que más influyen en la determinación de la PVB son el número y la profundidad de las perforaciones, ambas relacionadas con la posibilidad de disminuir la incertidumbre epistémica. El mismo valor de PVB es un factor que afecta la determinación de la incertidumbre. Entre más pequeño la PVB, mayor es la incertidumbre en su determinación.

\section{REFERENCIAS}

Baecher, G., and Christian, J. (2005). Reliability and statistics in geotechnical engineering. West Sussex: John Wiley \& Sons.

Colmenares, J., Dávila, J., Vega, J., and Shin, J. (2018). Tunnelling on terrace soil deposits: Characterization and experiences on the Bogotá-Villavicencio road. Geomechanics and Engineering, 15(3), 899-910. doi: 10.12989/ gae.2018.15.3.899.

Das, B. (2001). Fundamentos de ingeniería geotécnica. Sacramento: Thomson Learning.

Fernández, H., y Ferrando, F. (2018). Glaciares rocosos en la zona semiárida de Chile: relevancia de un recurso hídrico sin protección normativa. Cuadernos de Geografía: Revista Colombiana de Geografía, 27(2), 338-355. doi: 10.15446/rcdg. v27n2.63370.

Goodman, R., and Ahlgren, C. (2000). Evaluating safety of concrete gravity dam on weak rock: scott dam. Journal of Geotechnical and Geoenvironmental Engineering, 126(5), 429-442.

Kahraman, S., and Alber, M. (2006). Estimating unconfined compressive strength and elastic modulus of a fault breccia mixture of weak blocks and strong matrix. International Journal of Rock Mechanics and Mining Sciences, 43(8), 12771287. doi: 10.1016/j.ijrmms.2006.03.017.

Kahraman, S., Alber, M., Fener, M., and Gunaydin, O. (2015). An assessment on the indirect determination of the volumetric block proportion of Misis fault breccia (Adana, Turkey). Bulletin of Engineering Geology and the Environment, 74(3), 899-907. doi: 10.1007/s10064-014-0666-9.

Lindquist, E.S, and Goodman, R.E. (1994). Strength and deformation properties of a physical model melange. 1st North American Rock Mechanics Symposium. Austin, Texas.

Mahdevari, S., and Maarefvand, P. (2017). Applying ultrasonic waves to evaluate the volumetric block proportion of bimrocks. Arabian Journal of Geosciences, 10(9). doi: 10.1007/s12517-0172999-8. 
Manchego, A., y Peña, G. (2015). Estudio geológico y caracterización geotécnica en el sector rural de la vereda Helechal y Guasimal del municipio de Tena departamento de Cundinamarca. Tesis, Universidad Pedagógica y Tecnológica de Colombia, Colombia.

Medley, E. (1994). The engineering Characterization of Melanges and Similar Block in Matrix Rocks (Bimrocks). Ph.D. Thesis, University of California, USA.

Medley, E. (1997). Uncertainty in estimates of block volumetric proportions in melange bimrocks. International Symposium on Engineering Geology and the Environment. Athens, Greece.

Medley, E. (2002). Estimating block size distributions of melanges and similar block-in-matrix rocks (bimrocks). 5th North American Rock Mechanics Symposium. Toronto, Canada.

Medley, E., and Goodman, R. (1994). Estimating the block volumetric proportion of melanges and similar block-in-matrix rocks (bimrocks). $1 s t$ North American Rock Mechanics Symposium. Austin, Texas.

Napoli, M., Barbero, M., Ravera, E., and Scavia, C. (2018). A stochastic approach to slope stability analysis in bimrocks. International Journal of Rock Mechanics and Mining Sciences, 101, 4149. doi: 10.1016/j.ijrmms.2017.11.009.

Reneau, S., and Dietrich, W. (1991). Erosion rates in the southern Oregon Coast Range: Evidence for an equilibrium between hillslope erosion and sediment yield. Earth Surface Processes and Landforms, 16(4), 307-322. doi: 10.1002/ esp.3290160405.

Sonmez, H., Gokceoglu, C., Tuncay, E., Medley, E., and Nefeslioglu, H. (2004). Relationships between volumetric block proportions and overall UCS of a volcanic bimrock. FELSBAU - Rock and Soil Engineering - Journal for Engineering Geology, Geomechanics and Tunneling, 22(5), 27-34.

Sonmez, H., Gokceoglu, C., Medley, E., Tuncay, E., and Nefeslioglu, H. (2006). Estimating the uniaxial compressive strength of a volcanic bimrock. International Journal of Rock Mechanics and Mining Sciences, 43(4), 554-561. doi: 10.1016/j. ijrmms.2005.09.014.

Tien, Y., Lu, Y., Chung, Y., Wu, T., Lin, J., Kou, M., and Lee, D. (2010). Uncertainty in estimation of volumetric block proportion of bimrocks by using scanline method. 44th U.S. Rock Mechanics Symposium and 5th U.S.-Canada Rock Mechanics Symposium. Utah, USA.

Tien, Y., Lu, Y., Wu, T., Lin, J., and Lee, D. (2011). Quantify uncertainty in scanline estimates of volumetric fraction of anisotropic bimrocks. 45th U.S. Rock Mechanics/Geomechanics Symposium. California, USA.

Xu, W., Hu, R., and Tan, R. (2007). Some geomechanical properties of soil-rock mixtures in the Hutiao Gorge area, China. Geotechnique, 57(3), 255-264.

Alfonso Mariano Ramos-Cañón

ORCID: 0000-0003-2306-4333

Lady Carolina Castro-Malaver

ORCID: 0000-0001-6785-6848

Nelly Vanessa Padilla-Bello

ORCID: 0000-0002-6030-1986

Carlos Alberto Vega-Posada ORCID: 0000-0002-0923-356X

Trabajo recibido: octubre 24 de 2018

Trabajo aceptado: noviembre 07 de 2019 